\title{
LIE ALGEBRA COHOMOLOGY OF CERTAIN INFINITE-DIMENSIONAL REPRESENTATIONS
}

\author{
BY M. S. OSBORNE
}

\section{Communicated April 15, 1974}

Let $G$ denote a connected semisimple Lie group, $\mathfrak{g}$ its Lie algebra, $\mathfrak{g}_{c}$ the complexification of $\mathfrak{g}$, and $\sigma$ the conjugation of $\mathfrak{g}_{c}$ with respect to $\mathfrak{g}$. The Lie algebra cohomology of certain nilpotent subalgebras of $\mathfrak{g}_{c}$ plays an important role in the Bott-Borel-Weil theorem and its generalizations, notably in the works of Kostant [1], Schmid [3], and others. An important consideration is the regular behavior of the group actions associated to the Lie algebra cohomology complex.

In this note we announce some results concerning the case where the nilpotent subalgebra mentioned above comes from an Iwasawa decomposition of $\mathrm{g}$. This case is virtually the opposite of that considered by Schmid, and the aforementioned regularity is only partial.

I would like to thank my thesis advisor, Robert Langlands, for his encouragement and generous advice.

1. The setting. Let $\mathfrak{g}=\mathfrak{f}+\mathfrak{p}$ be a Cartan decomposition of $\mathfrak{g}, \theta$ the corresponding Cartan involution, i a $\theta$-stable Cartan subalgebra of $\mathfrak{g}$, $\dot{\mathfrak{i}}_{c}$ the complexification of $\mathfrak{i}, \mathfrak{b}_{c}$ a Borel subalgebra of $\mathfrak{g}_{c}$ containing $\mathfrak{j}_{c}$, and $\mathfrak{p}_{c}$ a parabolic subalgebra of $\mathfrak{g}_{c}$ containing $\mathfrak{b}_{c}$. Let $\mathfrak{n}_{c}^{+}$denote the nil radical of $\mathfrak{p}_{c} ; \mathfrak{n}_{c}^{+}$will be used in computing cohomology. Let $\mathfrak{l}_{c}$ be a $\sigma$-stable Levi factor of $\mathfrak{p}_{c}$, and set $\mathfrak{l}=\mathfrak{l}_{c} \cap \mathfrak{g}$. Let $L$ denote the centralizer in $G$ of the center of $\mathfrak{I} ; L$ is then a reductive subgroup of $G$ which normalizes $\mathfrak{n}_{c}^{+}$. In the work of Kostant, $G$ is compact. In that of Schmid, $\mathfrak{p}_{c}=\mathfrak{b}_{c}$ and $\mathfrak{j} \subset \mathfrak{f}$. In the work of the author, $\mathfrak{p}_{c}$ is the complexification of a minimal parabolic subalgebra of $\mathfrak{g}$, so that $\mathrm{n}_{c}^{+}$is the complexification of the nilpotent constituent of an Iwasawa decomposition of $\mathfrak{g}$.

Let $\pi$ be a representation of $G$ in a Hilbert space $\mathscr{H}$, and assume that the Jordan-Hölder series of $\mathscr{H}$ is finite. Let $\mathscr{H}_{\infty}$ denote the space of $C^{\infty}$-vectors in $\mathscr{H}$. One may form the Lie algebra cohomology complex $\left(C^{q}\left(\mathfrak{n}_{c}^{+}, \mathscr{H}_{\infty}\right), d^{q}\right)$ and its associated cohomology spaces $H^{q}\left(\mathfrak{n}_{c}^{+}, \mathscr{H}_{\infty}\right)$. Since $\mathscr{H}_{\infty}$ is a Fréchet space, so is $C^{q}\left(\mathfrak{n}_{c}^{+}, \mathscr{H}_{\infty}\right)$, and the differentials $d^{q}$ are continuous linear maps. $\left(n_{c}^{+}, \pi\right)$ will be called a CF-pair (for closed range, finite dimension) if each $d^{q}$ has closed range and each $H^{q}\left(\mathfrak{n}_{c}^{+}, \mathscr{H}_{\infty}\right)$ is finite dimensional.

AMS (MOS) subject classifications (1970). Primary 18H25, $22 \mathrm{E} 45$. 
2. Traces. If $f \in C_{c}^{\infty}(G), \pi(f)$ is trace class on $\mathscr{H}$, and

$$
\operatorname{trace}[\pi(f)]=\int_{G} f(g) \Theta_{\pi}(g) d g,
$$

where the character $\Theta_{\pi}$ of $\pi$ is locally integrable and analytic on the regular set $G^{\prime}$ in $G$. Thus one may define an extended trace, $\operatorname{trace}^{E}[\pi(T)]=T\left(\Theta_{\pi}\right)$ for any compactly supported Radon measure $T$ on $G^{\prime}$. One can show without difficulty that if $\pi(T)$ is actually trace class, then its trace is $T\left(\Theta_{\pi}\right)$ (and, as a corollary, that if $\pi(T)=\pi\left(T^{\prime}\right)$, then $T\left(\Theta_{\pi}\right)=T^{\prime}\left(\Theta_{\pi}\right)$ ). Thus $\operatorname{trace}^{E}[\pi(T)]$ depends only on $\pi(T)$ and is a legitimate extension of the trace map. In this context, $\operatorname{trace}^{E}[\pi(g)]=\Theta_{\pi}(g)$ if $g \in G^{\prime}$.

If $g \in L$, then $g$ acts on $C^{q}\left(\mathfrak{n}_{c}^{+}, \mathscr{H}_{\infty}\right)$ via $\pi^{q}(g)$, and on $H^{q}\left(\mathfrak{n}_{c}^{+}, \mathscr{H}_{\infty}\right)$ via $\pi^{q *}(g)$. In view of earlier conventions, one may define for $g \in L^{\prime}=$ $L \cap G^{\prime}$ :

$$
\operatorname{trace}^{E}\left[\pi^{\alpha}(g)\right]=\operatorname{trace}^{E}[\pi(g)] \operatorname{trace}\left[\left.\operatorname{Ad}\left(g^{-1}\right)\right|_{\Lambda^{q} \mathfrak{n}_{c}^{+}}\right] .
$$

Thus if $D(g)=\operatorname{det}\left(1-\left.\operatorname{Ad}\left(g^{-1}\right)\right|_{\mathfrak{n}_{c}^{+}}\right)$, then

$$
\sum_{\alpha}(-1)^{q} \operatorname{trace}^{E}\left[\pi^{q}(g)\right]=D(g) \Theta_{\pi}(g) .
$$

Let $S \subset L^{\prime}$. Call $\left(\mathrm{n}_{c}^{+}, \pi, S\right)$ an AT-triple (for alternating sum of traces) if $\left(\mathrm{n}_{c}^{+}, \pi\right)$ is a CF-pair, and for all $g \in S$,

$$
D(g) \Theta_{\pi}(g)=\sum_{\alpha}(-1)^{\alpha} \operatorname{trace}\left[\pi^{\alpha *}(g)\right] .
$$

Note that if $\mathscr{H}$ is finite dimensional, then $\left(\mathfrak{n}_{c}^{+}, \pi, L^{\prime}\right)$ is always an AT-triple.

3. Results. Now assume that $\mathfrak{p}_{c}$ is the complexification of a minimal parabolic subalgebra of $\mathfrak{g}$. In what follows, the term "principal series" will refer to the full (unitary or nonunitary) principal series of $G$.

Note first that if $\mathrm{n}_{c}^{+}$is odd dimensional, and $\pi$ is any principal series representation, then $\left(\mathfrak{n}_{c}^{+}, \pi, L^{\prime}\right)$ is never an AT-triple. This is because $|D(g)| \Theta_{\pi}(g)$ extends to an analytic function on $L$, but with $n_{c}^{+}$odd dimensional, $D(g)$ assumes both positive and negative values, whence $D(g) \Theta_{\pi}(g)$ has no analytic extension to $L$. On the other hand, the right-hand side of (1) is analytic on $L$ if $\left(\mathfrak{n}_{c}^{+}, \pi\right)$ is a CF-pair.

Let $S=\left\{g \in L^{\prime} \mid \operatorname{det}\left(1-\left.\operatorname{Ad}(g)\right|_{\mathfrak{n}_{c}^{+}}\right)>0\right\}$.

Theorem A. Let $G=\operatorname{SL}(2, R)$ or $\operatorname{SL}(2, C)$. If $\pi$ is any irreducible unitary or principal series representation of $G$, then $\left(n_{c}^{+}, \pi, S\right)$ is an $A T$ triple. 
TheOREM B. Let $G$ be a finite covering group of $S O_{e}(1, p), p \geqq 2$. There exist at most countably many distinct principal series representations $\pi$ of $G$ such that $\left(\mathrm{n}_{c}^{+}, \pi, S\right)$ is not an $A T$-triple.

REMARKS. Of course, there are a continuum of distinct principal series representations of $G$, so Theorem B is not vacuous. In all cases, $d^{q}$ has closed range, and $\left(\mathfrak{n}_{c}^{+}, \pi, S\right)$ is AT if and only if $H^{q}\left(\mathfrak{n}_{c}^{+}, \mathscr{H}_{\infty}\right)$ is finite dimensional for all $q$. Whether this ever fails is not known to the author. Finally, if $p$ is odd, $S=L^{\prime}$.

In the situation of Theorem $\mathrm{B}$, the first and last cohomology spaces are finite dimensional; furthermore, $\sum(-1)^{q} \operatorname{dim} H^{q}\left(\mathfrak{n}_{c}^{+}, \mathscr{H}_{\infty}\right)$ is always finite. Hence the countable set in Theorem B must be empty when $G=\operatorname{SL}(2, R)$ or $\operatorname{SL}(2, \boldsymbol{C})$. Theorem A then follows from Theorem B by using the explicit form in which irreducible unitary representations arise inside the principal series.

Theorem B is proven by analyzing the inducing construction, and the techniques are primarily analytic: $\mathscr{H}_{\infty}$ is a space of $C^{\infty}$ functions on $G$, and can be broken up using the Bruhat lemma. The trace formula (1) is proven by explicitly evaluating both sides and observing that they are equal.

There are other spaces that can be used for cohomological purposes. For example, if $\mathscr{H}_{K}$ is the space of $K$-finite vectors, then $H^{q}\left(\mathfrak{n}_{c}^{+}, \mathscr{H}_{K}\right)$ is always finite dimensional. These spaces have been studied by Casselman (unpublished). The structure of $H^{q}\left(\mathrm{n}_{c}^{+}, \mathscr{H}_{K}\right)$ has consequences for $H^{q}\left(\mathfrak{n}_{c}^{+}, \mathscr{H}_{\infty}\right)$, but there are limitations to the use of $\mathscr{H}_{K}$ due to the fact that $\mathscr{H}_{K}$ is not $G$-invariant.

Finally, the constructions of Schmid and others fit into the situation described above. Details will be published at a future date.

\section{REFERENCES}

1. B. Kostant, Lie algebra cohomology and the generalized Borel-Weil theorem, Ann. of Math. (2) 74 (1961), 329-387. MR 26 \#265.

2. M. S. Osborne, Lefschetz formulas on non-elliptic complexes, Thesis, Yale University, New Haven, Conn., 1972.

3. W. Schmid, On a conjecture of Langlands, Ann. of Math. (2) 93 (1971), 1-42. MR 44 \#4149.

4. G. Warner, Harmonic analysis on semisimple Lie groups. I Die Grundlehren der math. Wissenschaften, Band 188, Springer-Verlag, New York, 1972.

Department of Mathematics, University of Chicago, Chicago, Illinois 60637 